\title{
Der Lehrberuf im Wandel? - Über Grenzen von Leadership
}

\section{Bruno Leutwyler und Priska Sieber}

Die in den vergangenen Jahren zu beobachtende Weitergabe von Verantwortung an die Schulen ist in den meisten Schweizer Kantonen mit der Einführung von Schulleitungen einhergegangen. Damit wurde in Ansätzen möglich, was in anderen Ländern bereits seit Längerem zum Berufsbild von Lehrpersonen gehört: eine berufliche Laufbahn. Allerdings sind bisherige Versuche, in der Schweiz weitere berufliche Aufstiegsmöglichkeiten oder Rollendifferenzierungen im Lehrberuf zu etablieren, meist auf heftige Abwehrreaktionen seitens der Lehrerschaft gestossen und hielten in der Folge auch Berufsverbände und Behörden davon ab, sich auf solche Konzepte ernsthaft einzulassen. Vor dem Hintergrund veränderter Rahmenbedingungen hat ein Forschungsprojekt des IZB nun untersucht, wie verschiedene Anspruchsgruppen heutzutage zu einer Einführung von Laufbahnmodellen stehen und wie weit in der Vergangenheit beobachtete Abwehrhaltungen heute noch verbreitet sind. Mittels Fragebogen wurden in vier Deutschweizer Kantonen Lehrkräfte verschiedener Volksschulstufen befragt. Zudem wurden vertiefende Interviews mit Lehrpersonen und mit Vertretungen von Verwaltungen und Verbänden geführt. Die Ergebnisse der Studie zeigen, dass eine überragende Mehrheit der befragten Lehrpersonen eine Einführung von Laufbahnkonzepten grundsätzlich befürwortet, dass allerdings mit Laufbahnmodellen sehr unterschiedliche Hoffnungen verbunden werden. Die Befunde werfen die Frage auf, wie weit pädagogisches Leadership in der vorherrschenden Berufskultur gelebt werden kann, und sie machen deutlich, dass Lehrpersonen durchaus gewillt sind, sich selbst am Leadership aktiv zu beteiligen.

In den vergangenen Jahren wurden im Rahmen neuer Steuerungsmodelle den Schulen erweiterte Verantwortungen übertragen, um auf die zunehmende Komplexität der Wirklichkeit und der damit verbundenen gegenwärtigen und künftigen erzieherischen Herausforderungen flexibel und lokal angepasst reagieren zu können. Die Einführung der neuen Steuerungsmodelle ist in den meisten Schweizer Kantonen mit der Implementierung von Schulleitungen einhergegangen, die Leadership bezüglich der jeweiligen lokalen Schuleinheiten mit deren neu übertragenen Aufgaben übernehmen sollen. 
Mit der Einführung von Schulleitungen wurde in Ansätzen möglich, was in anderen Berufen eine Selbstverständlichkeit ist und in einigen Ländern zum Berufsbild von Lehrpersonen gehört: eine berufliche Laufbahn (Peske, Liu, Johnson, Kauffmann \& Kardos, 2001; Santiago, 2002; Schacter et al., 2003). Was in diesem Beitrag unter beruflichen Laufbahnen verstanden wird und welche Rolle Möglichkeiten und Formen von Laufbahnmodellen in der Diskussion um Leadership spielen, wird in einem ersten Abschnitt dieses Beitrags ausgeführt.

Allerdings sind bisherige Überlegungen in Richtung einer Rollen- bzw. Funktionsdifferenzierung der Lehrerschaft in der Schweiz insbesondere bei den Lehrpersonen selber auf teilweise heftige Ablehnung gestossen. Ein kurzer Abriss über diesen Diskurs wird in einem zweiten Abschnitt dargestellt. Damit soll darauf verwiesen werden, dass eine kritische Betrachtung von Laufbahnmodellen im Lehrberuf angemessen ist, da sie sowohl Grundfragen des Lehrerbildes und des Professionsverständnisses (Ender \& Strittmatter, 2001; Hearvey-Beavis, 2003), als auch Fragen bezüglich Arbeitsbedingungen betreffen. Beides sind zentrale Themen des Dachverbandes Schweizer Lehrerinnen und Lehrer (LCH), dessen erklärtes Ziel ist, zur Attraktivität und zum Ansehen des Lehrberufs sowie zur Verbesserung der Arbeitsbedingungen der Lehrkräfte beizutragen. In diesem Zusammenhang fordert der LCH in seinem Berufsleitbild: «Lehrpersonen verstehen ihre unterschiedlichen Fähigkeiten und Interessen als Ressource und Bereicherung. Sie entwickeln Perspektiven für ihren Arbeitsplatz und ihre berufliche Laufbahn» (LCH, 1999, S. 19). ${ }^{1}$

Vor diesem Hintergrund stellt sich deshalb die Frage, ob Lehrpersonen überhaupt bereit wären, unterschiedliche Rollen und Funktionen zu übernehmen und die damit einhergehende Funktionsdifferenzierung zu akzeptieren. Entsprechend hat der LCH eine "Untersuchung zur Akzeptanz von Laufbahnmodellen im Lehrberuf - Chancen- und Risikoeinschätzung» in Auftrag gegeben, die vom Institut für internationale Zusammenarbeit in Bildungsfragen (IZB) der Pädagogischen Hochschule Zentralschweiz (PHZ), Teilschule Zug, in der ersten Hälfte des Jahres 2005 durchgeführt wurde (Leutwyler, Sieber \& Diebold, 2005). Im dritten Abschnitt wird kurz das Gesamtkonzept dieser Untersuchung skizziert und die zentralen Fragen sowie das methodische Vorgehen bezüglich des in diesem Beitrag präsentierten Teilaspekts der Studie vorgestellt, einer schriftlichen Befragung von Lehrpersonen in vier Deutschschweizer Kantonen.

Die Ergebnisse dieser Befragung werden im vierten Abschnitt präsentiert. Daran schliessen Interpretationen der Befunde an (Abschnitt 5), bei denen - um es vorweg zu nehmen - eine ganze Reihe weiterer Fragen aufgeworfen werden. Sie betreffen u.a. das Problem der Rolle der Schulleitungen als Leader sowie Fragen im Zusammenhang mit erweiterten Tätigkeitsbereichen von Lehrpersonen, für die ein Teil der Lehrerschaft bereit wäre, eine Leadershipfunktion zu übernehmen. 


\section{Laufbahnmodelle im Lehrberuf}

Eine berufliche Laufbahn bezeichnet allgemein die Gesamtheit der Stellenfolgen einer Person während ihres beruflichen Lebenslaufs. Damit sind Berufsentwicklungen angesprochen (entsprechend der englischen Bezeichnung "career»), zu denen sowohl hierarchisch vertikale Positionsfolgen als auch horizontale Stellenwechsel innerhalb einer Organisation und zwischen Organisationen zählen.

Diese allgemeine Definition macht deutlich, dass berufliche Laufbahnen aus zwei verschiedenen Perspektiven von Bedeutung sind. Aus der Sicht der Mitarbeitenden ist eine berufliche Laufbahn Ausdruck je unterschiedlicher persönlicher Bedürfnisse, Fähigkeiten und Ziele. Durch die Wahl von bestimmten Arbeitsumwelten versuchen Personen diese zu befriedigen bzw. umzusetzen. Aus der Sicht der Organisation resp. der Schule beziehen sich Laufbahnmodelle auf die Gestaltung von Stellen und Positionen, die einerseits in ihrer Gesamtheit zu einer optimalen Erfüllung der anstehenden Aufgaben durch zufriedene Mitarbeitende führen und anderseits qualifizierte und engagierte Mitarbeitende anzuziehen und zu halten vermögen (Leutwyler et al., 2005).

Institutionalisierte Laufbahnstufen oder -abschnitte definieren so von der Organisation oder von der Profession erwünschte, angestrebte berufliche Entwicklungen. Solche erwünschten beruflichen Entwicklungen entsprechen höheren Qualifikationen, die sich erst aufgrund von beruflicher Erfahrung oder aber aufgrund von Weiterbildung ergeben. Laufbahnmodelle sind so eine Form, um Erwartungen, Anforderungen und Möglichkeiten beruflicher Entwicklungen zu institutionalisieren (Seifert, 1992, S. 194).

Bis vor kurzem kannte der Lehrberuf in der Schweiz kaum unterschiedliche Funktionen und keine Aufstiegsmöglichkeiten. Lehrerinnen und Lehrer traten nach der Grundausbildung in den Unterrichtsberuf ein und blieben als «Lehrerinnen und Lehrer» in dieser Tätigkeit bis zu ihrer Pensionierung. Für ihre dabei gemachten arbeitsbezogenen Erfahrungen und Entwicklungen gab es berufsintern keine Stellen mit erweiterten Aufgaben und Verantwortungen zu vergeben und alle Lehrpersonen wurden für ihre je unterschiedlichen Entwicklungen gleichermassen belohnt, indem mit zunehmendem Dienstalter auch die finanzielle Vergütung für ihre Arbeit anstieg.

Laufbahnmodelle im Lehrberuf - wie sie im vorliegenden Beitrag definiert sind - beschreiben demgegenüber die Möglichkeit einer berufsinternen Abfolge von Stellen, wonach für besondere Kompetenzentwicklungen von Lehrpersonen entsprechende Positionen bereitgestellt werden, die sowohl mit einer Veränderung von Rollen bzw. Funktionen ${ }^{2}$ mit besonderen Aufgaben und Verantwortlichkeiten verbunden sind - also beispielsweise auch Leadershipfunktionen -, als auch mit einer entsprechenden Anerkennung der damit verbundenen Kompetenzen einhergehen.

Die Idee, Laufbahnmodellen im Lehrberuf in Erwägung zu ziehen, wird von verschiedenen gegenwärtigen Entwicklungen gestützt (Leutwyler et al., 2005). 
Insbesondere im Zusammenhang mit den aktuellen Diskussionen um Schulleitungen bzw. Leadership erhalten Möglichkeiten und Formen von Laufbahnmodellen eine besondere Rolle.

Zum einen wurden im Rahmen der bereits genannten neuen Steuerungsmodelle in den meisten Schweizer Kantonen Schulleitungen implementiert, die Leadership bezüglich der jeweiligen lokalen Schuleinheiten mit deren neu übertragenen Aufgaben zu übernehmen haben. Damit wurde eine Laufbahnstufe im Lehrberuf und so gewissermassen die Möglichkeit einer Führungslaufbahn geschaffen. Teilweise sind auch weitere hierarchische Stufungen dazu gekommen: Schulhausleitungen, Stufenleitungen etc., auch Stellen mit Spezialfunktionen wie beispielsweise Informatikverantwortliche. Im Schulbereich «Karriere zu machen», ist in einem eingeschränkten Rahmen nun möglich geworden. Doch ein beruflicher Aufstieg bedeutet in der Regel noch immer ein entsprechendes Aussteigen aus der Unterrichtstätigkeit, wodurch gerade leistungsorientierte Lehrpersonen dem Unterricht «verloren» gehen; das gegenwärtige System tendiert dazu, initiative Persönlichkeiten mit überdurchschnittlichem Einsatz und hoher Kompetenz aus dem Schulzimmer oder gar aus dem Schuldienst zu verdrängen. Theoretisch sind demgegenüber auch Laufbahnmodelle denkbar, die eine berufliche Entwicklung innerhalb der Unterrichtstätigkeiten erlauben. In den Organisationswissenschaften wird die Möglichkeit, für Spezialistinnen und Spezialisten unterschiedliche Positionen bereit zu halten, ohne sie zu Führungskräften machen zu müssen, mit dem Begriff der «Fachlaufbahn» bezeichnet (Domsch, 1994; Füchtner, 1998).

Zum anderen zeigen aktuelle theoretische und empirische Auseinandersetzungen um Leadership in Schulen, dass erfolgreiches Leadership mehr beinhaltet als die Aktivitäten von Schulleitungen. Gut geführten Schulen, die das Lernen der Schülerschaft in besonderem Masse fördern, steht nicht ein "Superhero» (Education Writers Association, 2002) oder ein «know-it-all» (Spillane, 2005) vor. Effektive Konzepte von Leadership folgen eher der Vor-stellung von «Distributed Leadership» (Bennett, Wise, Woods \& Harvey, 2003). Darunter wird ein Modell von Leadership verstanden, bei dem mög-lichst alle in einer Organisation verfügbaren menschlichen Potenziale und Expertisen freigesetzt werden und entsprechend Leadership rekonzeptualisiert wird als Leadership "of many rather than the few» (Harris \& Lambert, 2003). Implizit in einem solchen Modell von Leadership sind die Leadership-Praktiken von Lehrpersonen, wobei diese grundsätzlich als informelle Leaders, aber auch in formellen LeadershipRollen beispielsweise als Abteilungsleitende, Fachkoordinierende oder Mentorinnen, Mentoren von Lehrpersonen fungieren (Muijs \& Harris, 2003). Laufbahnmodelle im Lehrberuf verfügen entsprechend über das Potenzial, Leadership innerhalb einer Organisation breiter zu verteilen, insbesondere wenn Schulleitende dem Zugang von Distributed Leadership folgen und ihre Rolle als primär darin verstehen, andere an der Schule Beteiligte zu befähigen zu führen (Harris, 2004). 
Es bestehen also gute Gründe, sich mit Laufbahnmodellen im Lehrberuf auseinanderzusetzen. Allerdings sind Überlegungen in diese Richtung in der Vergangenheit auf teilweise heftige Ablehnung gestossen. Entsprechende Erfahrungen werden im folgenden Abschnitt zusammengefasst.

\section{Erfahrungen mit bisherigen Laufbahnkonzepten in der Schweiz}

Lehrerlaufbahnen oder zumindest Veränderungen der Rollen und Funktionen von Lehrpersonen im Laufe ihres Berufslebens wurden schon in früheren Reflexionen und Untersuchungen zum Lehrberuf in der Schweiz thematisiert.

Bereits Mitte der 1970er Jahre wurden im Bericht der Expertenkommission «Lehrerbildung von morgen» (Müller, 1975) die Notwendigkeit und Grenzen einer Spezialisierung von Lehrkräften thematisiert. «Die Spezialisierung versucht [...], einerseits die Begabung des einzelnen Lehrers weiterzuentwickeln und anderseits die notwendigen Funktionen im Schulsystem mit geeigneten Leuten zu besetzen» (ebd., S. 57). Die Autoren schlagen in ihrem Bericht eine «strukturierte Lehrerschaft» vor mit stufen-, fach- und funktionsspezifischen Rollendifferenzierungen. Für neu zu übernehmende Rollen sollen sich Lehrpersonen in Weiterbildungskursen Spezialqualifikationen aneignen, ihre Funktionen anschliessend in klar umrissenen Gruppen ausüben und je nach Funktion seien für Spezialistinnen, Spezialisten auch Stundenentlastungen vorzusehen. Durch die Eröffnung von Spezialistenrollen bzw. Aufstiegsmöglichkeiten innerhalb des Lehrberufs bzw. einer Schulstufe wurde eine gesündere Bewältigung des komplexer werdenden Bildungsauftrags der Schulen, die Reduktion des Einzelkämpfertums bei Lehrpersonen sowie das Halten von weiterbildungs- und aufstiegsorientierten Lehrkräften erwartet.

Zwei neuere Beispiele der Auseinandersetzung mit Entwicklungen im Lehrberuf zeigen, dass das Thema Lehrerlaufbahnen nach wie vor als bedeutend eingeschätzt wird. ${ }^{3}$ Im Jahr 1999 hat die Arbeitsgruppe Lehrerbildung der EDK eine Erhebung zum «Bedarf an Zusatzausbildungen für Funktionen im Bildungssystem» in Auftrag gegeben, in der das Thema "Personalentwicklung und Weiterbildung» als weitaus wichtigstes Thema für die Schule beurteilt wird (Baumgartner \& Kassis, 2001). Ein zweites aktuelles Beispiel, das explizit Hierarchien im Lehrberuf aufgreift, entstand im Rahmen eines Auftrags der Task Force «Lehrberufsstand» der EDK (Bucher \& Nicolet, 2003). Darin wird die Einführung der Rolle des «master teacher» resp. der Stamm-Lehrperson vorgeschlagen (ebd., S. 67-69).

Die Reaktionen auf diese Vorschläge fielen bei unterschiedlichen Anspruchsgruppen allerdings sehr verschieden aus. Die Literatur über frühere Diskussionen (EDK, 1978) sowie Interviews mit drei in entsprechenden Entwicklungsprojekten involvierten Fachleuten (Leutwyler et al., 2005) weisen darauf hin, dass in der Vergangenheit grob drei unterschiedliche Reaktionsmuster beschrieben werden können. 
1. Die EDK begegnete Vorschlägen in Richtung einer differenzierten Lehrerschaft und der Schaffung unterschiedlicher Positionen im Lehrberuf mit Interesse, empfahl entsprechende Modelle in der Regel zur weiteren Prüfung und vergab verschiedentlich neue Aufträge, die u.a. Denkanstösse in diese Richtung lieferten (vgl. oben).

2. Die kantonalen Bildungsverwaltungen beurteilten eine solche Weiterentwicklung des Lehrberufs zwar als zeitgemäss und grundsätzlich attraktiv für den Lehrberuf, doch waren sie bezüglich einer konkreten Umsetzung zurückhaltend, weil sie die Kosten vor den antizipierten organisatorischen Zusatzaufwendungen scheuten.

3. Die Lehrerschaft bzw. die Lehrervereinigungen reagierten demgegenüber vornehmlich mit einer dezidiert ablehnenden Haltung. Im Auswertungsbericht zur Vernehmlassung des Berichts der Expertenkommission «Lehrerbildung von morgen» wurden als befürchtete Wirkungen eines entsprechenden Modells eine «Diplomhascherei», eine einseitige Spezialisierung und die Schaffung von Hierarchien in der Lehrerschaft kritisiert (EDK, 1978).

Mit Spezialisierung und Hierarchisierung sind zwei wichtige Dimensionen einer «strukturierten Lehrerschaft», von Laufbahnmodellen im Lehrberuf bzw. von Distributed Leadership angesprochen, die eine einschneidende Veränderungen im beruflichen Selbstverständnis der Profession bewirken würden, da sie dem im Lehrberuf traditionell tief verankerten Gleichheitsprinzip widersprechen. Aus theoretischer Perspektive kann somit die Ablehnung von Laufbahnmodellen seitens der Lehrkräfte mit deren Orientierung am Gleichheitsprinzip erklärt werden, das in der einschlägigen Literatur zum Lehrberuf häufig nach dem einflussreichen Aufsatz von Lortie (1972) als «Autonomie-Paritäts-Muster» bezeichnet wird. ${ }^{4}$

\section{Konzept und Methoden der Studie}

Vor diesem Hintergrund beauftragte der LCH das IZB, Grundlagen für die Einschätzung der Wünschbarkeit von Laufbahnmodellen im Lehrberuf zu erarbeiten. Das IZB untersuchte einerseits intendierte und auch nicht beabsichtigte Folgen von Laufbahnmodellen, indem es Fallstudien in Kontexten und in Ländern durchführte, in denen Laufbahnmodelle umgesetzt wurden (Leutwyler et al., 2005). Andererseits erhob es persönliche Einstellungen und Erwartungen im Zusammenhang mit Laufbahnmodellen bei verschiedenen Anspruchsgruppen im schweizerischen Kontext über schriftliche und mündliche Befragungen. Für die hier referierte Fragestellung, wie weit Lehrpersonen in der Schweiz heutzutage bereit sind, unterschiedliche Funktionen in den Schulen zu übernehmen und sich so aktiv am Leadership zu beteiligen, werden ausschliesslich die Resultate der schriftlichen Befragung von Lehrpersonen berichtet. Dabei interessierte insbesondere, wie amtierende Lehrpersonen zu einer allfälligen Einführung von Laufbahnmodellen stehen, wie weit in der Vergangenheit beobachtete Abwehr- 
haltungen heute verbreitet sind und wovon eine allfällige Akzeptanz bei den Lehrpersonen abhängig ist.

In die schriftliche Befragung einbezogen wurden Lehrpersonen aus vier Kantonen. Die Auswahl der Kantone erfolgte aufgrund theoriegeleiteter Kriterien: Da eine Einführung von Laufbahnmodellen das traditionelle Gleichheitsprinzip in der Lehrerschaft in Frage stellen und über neue Zusammenarbeits- und Wissenstransfer-Formen ein anderes Autonomie-Verständnis bedingen würde, wurden Kantone in die Stichprobe aufgenommen, die diesbezüglich unterschiedliche Vorerfahrungen aufweisen. So wurden Kantone ausgewählt, die einerseits mit der Einführung von Schulleitungen, die mit Personalführungskompetenzen ausgestattet sind, das zentrale Paritäts-Prinzip missachten, sowie andererseits mit der Einführung von Qualitätsentwicklungsmassnahmen in den Schulen (wie bspw. Selbstevaluation der einzelnen Schulen) das traditionelle Autonomie-Prinzip in Frage stellen. Es wurden Kantone, die diesbezüglich sehr weitgehende Reformen initiiert haben, solchen Kantonen gegenübergestellt, die noch kaum oder erst vor kurzer Zeit entsprechende Schulentwicklungsvorhaben lanciert haben. Zudem wurden verschiedene Vorerfahrungen mit unterschiedlichen Qualifikationssystemen beachtet. Aufgrund dieser Kriterien wurden die Kantone Aargau, Appenzell-Innerrhoden, Luzern und St. Gallen in die Stichprobe einbezogen. Pro Kanton wurden je 200 Lehrpersonen aus der LCH-Mitgliederkartei gezogen. Die Gesamtpopulation bildeten also die Mitglieder des LCH der vier ausgewählten Kantone, wobei im LCH 95\% aller Volksschullehrpersonen der Deutschschweiz zusammengeschlossen sind.

Als Erhebungsinstrument wurde ein Fragebogen eingesetzt, der neben den zentralen Fragen zur Akzeptanz von Laufbahnmodellen Items oder Skalen zu folgenden Aspekten enthielt, bei denen ein Zusammenhang mit Einstellungen zu Laufbahnmodellen angenommen werden kann:

- Geschlecht, Alter, Pensum, Funktion, Berufserfahrung und Unterrichtsstufe der Lehrpersonen

- Berufsspezifische Einstellungen: eigene Skalen in Anlehnung an das Autonomie-Paritäts-Muster (Lortie, 1975) unter Einbezug von Items von Schwarzer und Jerusalem (1999);

- Engagement der Lehrpersonen: Skalen zur subjektiven Bedeutsamkeit der Arbeit, zur Verausgabungsbereitschaft, zum Ausmass der inneren Kündigung und zum beruflichen Ehrgeiz in Anlehnung an Schaarschmidt und Fischer (1996) sowie eine eigene Skala zum relativen Engagement im Vergleich zu den Kolleginnen und Kollegen;

- Berufszufriedenheit und Arbeitsmotivation: eigene Skalen (in Anlehnung an Ammann, 2004; Fiechter, Stienen \& Bühler, 2004);

- Merkmale der Schulen, in denen die Lehrpersonen arbeiten: Grösse; Kantonszugehörigkeit; Stand bezüglich konkreter Schulentwicklungsvorhaben (eigene Skala); kollektive Selbstwirksamkeitsüberzeugungen (Schwarzer \& Jerusalem, 1999); Kooperationskultur (Buholzer, 2000; Bessoth \& Landolt; 1997; Rolff, 1996). 
Die Erhebung wurde im April 2005 durchgeführt. Nach den üblichen Datenbereinigungsverfahren konnten von den insgesamt 800 verschickten Fragebögen 281 in die Auswertungen einbezogen werden. Dies entspricht einem Rücklauf von $35 \%$. In der Stichprobe sind die Frauen auf der Primarstufe mit $73.9 \%$ leicht übervertreten (vs. $70.6 \%$ in der Gesamtpopulation, Bundesamt für Statistik, 2000). Im Gegensatz dazu sind die Sekundarstufe I-Lehrerinnen mit 28.1\% in der Stichprobe deutlich untervertreten (vs. $40.4 \%$ in der Gesamtpopulation; vgl. ebd.). Aus diesem Grund wurden die Antworten für die deskriptiven Auswertungen entsprechend gewichtet.

\section{Ergebnisse}

Die schriftliche Befragung der Lehrpersonen zeigt deutlich, dass eine Mehrzahl der antwortenden Lehrpersonen bereit ist, unterschiedliche Rollen zu übernehmen und die damit einhergehende Funktionsdifferenzierung zu akzeptieren. So ist ein grosser Teil der Befragten der Ansicht, dass Lehrpersonen im Laufe ihrer beruflichen Entwicklung unterschiedlich viel Verantwortung übernehmen können $(80.0 \%)$ und dass nicht alle Lehrpersonen in einer Schule gleich viel Verantwortung zu übernehmen brauchen (76.6 \%). Fast alle Lehrpersonen (93.1\%) glauben denn auch, dass nicht alle Lehrpersonen im Laufe ihrer Berufslaufbahn ihre professionelle Kompetenz im gleichen Ausmass weiter entwickeln. Eine grosse Mehrheit der Lehrpersonen ist der Ansicht, dass diejenigen Lehrpersonen, die ihre professionelle Kompetenz überdurchschnittlich entwickeln, mehr Verantwortung in ihrer Schule (87.1 \%) und eine kommunizierbare Position mit mehr Verantwortung übernehmen können sollten $(85.7 \%)$ und dass sie besser bezahlt werden sollten $(75.7 \%)$.

\section{Akzeptanz von Laufbahnmodellen}

Eine Einführung von Laufbahnmodellen im Lehrberuf finden 88.8 \% der antwortenden Lehrpersonen sinnvoll. 5 Die Akzeptanz von Laufbahnmodellen unterscheidet sich jedoch stark je nach Alter $(96.2 \%$ der unter 30-Jährigen vs. $80.8 \%$ der über 50-Jährigen), nach Geschlecht (95.6\% der Lehrerinnen vs. 80.5 $\%$ der Lehrer) sowie nach Stufe (92.5 \% der Primarstufenlehrkräfte vs. $82.7 \%$ der Sekundarstufe I-Lehrpersonen). Junge Primarlehrerinnen akzeptieren Laufbahnmodelle also häufiger als ältere Sekundarlehrer.

Die Akzeptanz von Laufbahnmodellen unterscheidet sich auch je nach Ausprägung gewisser berufsspezifischer Merkmale. Zwar gibt es in Bezug auf das Ausmass an subjektiver Bedeutsamkeit der Arbeit und an innerer Kündigung keine systematischen Unterschiede in der Akzeptanz von Laufbahnmodellen, allerdings akzeptieren Lehrpersonen mit hohem beruflichem Ehrgeiz Laufbahnmodelle signifikant stärker als Lehrpersonen mit geringerem beruflichem Ehrgeiz (vgl. Tabelle 1). 
Ein ganz deutlicher Zusammenhang kann auch zwischen der Akzeptanz von Laufbahnmodellen und dem Ausmass festgestellt werden, wie stark sich Lehrpersonen mit dem Autonomie-Paritäts-Muster identifizieren. Lehrpersonen, die die Aspekte Autonomie und Parität in ihrer Arbeit stark gewichten, lehnen die Idee von Laufbahnmodellen hoch signifikant deutlicher ab als diejenigen, die diese Aspekte nicht so stark gewichten (vgl. Tabelle 1). Keine Zusammenhänge mit der Akzeptanz von Laufbahnmodellen können für die Motivation für den Lehrberuf und für Faktoren der Berufszufriedenheit nachgewiesen werden.

Tabelle 1: Mittelwerte (m) oder Häufigkeiten $(f)$ in Abhängigkeit von «Akzeptanz von Laufbahnmodellen (LBM)»

\begin{tabular}{|c|c|c|c|c|c|}
\hline & Variable & & Akzeptanz von & Akzeptanz von & $\chi^{2}$ - oder \\
\hline & & Aargau & $f=54$ & $f=7$ & \\
\hline 1 & Ko & Appenzell-Innerrhoden & $f=56$ & $f=7$ & $m^{2}(2)+2$ \\
\hline 1 & Kanton & Luzern & $f=61$ & $f=4$ & 2 \\
\hline & & St. Gallen & $f=68$ & $f=10$ & \\
\hline & & jünger als 30-jährig & $f=51$ & $f=2$ & \\
\hline 2 & Alter & 30- bis 49-jährig & $f=111$ & $f=10$ & $\chi^{2}(2)=9.025^{*}$ \\
\hline & & 50-jährig oder älter & $f=59$ & $f=14$ & \\
\hline 3 & Cecchble & männlich & $f=95$ & $f=23$ & \\
\hline 3 & Geschlecht & weiblich & $f=151$ & $f=7$ & $\chi^{2}($ \\
\hline 4 & Unterrichtsstufe & Primarstufe & $f=123$ & $f=10$ & $\gamma^{2}(1)-6705^{*}$ \\
\hline & & Sekundarstufe I & $f=81$ & $f=17$ & $\left.\chi^{-2}(1)=0.17\right)$ \\
\hline 5 & Beruflicher Ehrge & & $\mathrm{m}=3.10$ & $\mathrm{~m}=2.79$ & $\mathrm{t}=2.293^{*}$ \\
\hline 6 & Subjektive Bedeu & tsamkeit der Arbeit & $\mathrm{m}=3.51$ & $\mathrm{~m}=3.39$ & $\mathrm{t}=1.002$ \\
\hline 7 & Innere Kündigun & & $\mathrm{m}=2.22$ & $\mathrm{~m}=2.42$ & $\mathrm{t}=-1.302$ \\
\hline 8 & Autonomie-Parit & its-Muster & $\mathrm{m}=2.37$ & $\mathrm{~m}=2.87$ & $\mathrm{t}=6.230^{* * *}$ \\
\hline 9 & Kollektive Selbsty & virksamkeitsüberzeugungen & $\mathrm{m}=3.69$ & $\mathrm{~m}=3.36$ & $\mathrm{t}=2.338^{*}$ \\
\hline 10 & Kooperationskult & & $\mathrm{m}=2.56$ & $\mathrm{~m}=2.52$ & $\mathrm{t}=.347$ \\
\hline 11 & Stand der Schule & bzgl. Schulentwicklung & $\mathrm{m}=3.10$ & $\mathrm{~m}=2.90$ & $t=.744$ \\
\hline
\end{tabular}

Anmerkung: Chi-Quadrat für die Variablen ,Kanton', ,Alter', ,Geschlecht’ und ,Unterrichtsstufe'; t-Test für alle anderen Variablen. ${ }^{*} \mathrm{p}<.05 .{ }^{* *} \mathrm{p}<.01 .{ }^{* * *} \mathrm{p}<.001$.

Es stehen also insbesondere demographische und individuell-berufsspezifische Faktoren in einem Zusammenhang mit der Akzeptanz von Laufbahnmodellen. Schulspezifische Faktoren haben diesbezüglich kaum einen Einfluss. Lehrpersonen, die in hohem Ausmass auf die kollektive Problemlösefähigkeit in ihren Schulen vertrauen, akzeptieren Laufbahnmodelle zwar etwas häufiger als diejenigen mit einer geringen kollektiven Selbstwirksamkeitserwartung. Wenn aber nicht das Vertrauen in kollektive Problemlösefähigkeiten, sondern die Einschätzung der tatsächlichen Kooperationskultur der Schulen als Massstab für die 
Überwindung einer stark vereinzelten, segmentierten Arbeitskultur betrachtet wird, so ergeben sich keine Unterschiede im Hinblick auf die Akzeptanz von Laufbahnmodellen. Auch kein Einfluss auf die Akzeptanz von Laufbahnmodellen kann für den objektiven Stand der Schule bezüglich Schulentwicklungsvorhaben sowie für die Grösse der Schule nachgewiesen werden (vgl. Tabelle 1).

Es stehen also verschiedene Faktoren mit der Akzeptanz von Laufbahnmodellen in einem Zusammenhang. Mit binär logistischen Regressionen kann geschätzt werden, anhand welcher Einflussgrössen die Akzeptanz und die Ablehnung besonders gut unterschieden werden können, resp. mit welcher Wahrscheinlichkeit einzelne Faktoren eher zu einer Ablehnung von Laufbahnmodellen führen. In ein entsprechendes Regressionsmodell einzuführen sind als unabhängige Variablen diejenigen Faktoren, bei welchen sich signifikante Unterschiede bezüglich der Akzeptanz gezeigt haben. Entgegen den Erwartungen gehören die Kantone nicht zu diesen Variablen (vgl. Tabelle 1): Zwischen den vier ausgewählten Kantonen sind keine signifikanten Unterschiede bei der Akzeptanz von Laufbahnmodellen festzustellen. Aufgrund der bivariaten Verteilungen scheinen das Alter, das Geschlecht, die Unterrichtsstufe, der berufliche Ehrgeiz, das Ausmass der Identifikation mit dem Autonomie-Paritäts-Muster sowie die kollektiven Selbstwirksamkeitserwartungen in einem Zusammenhang mit der Akzeptanz zu stehen. In Tabelle 2 sind die Interkorrelationen zwischen der abhängigen und den unabhängigen Variablen aufgeführt.

\section{Tabelle 2: Interkorrelationen für «Akzeptanz von Laufbahnmodellen» und} Prädiktorvariablen

\begin{tabular}{llcccccc}
\hline Variable & 1 & 2 & 3 & 4 & 5 & 6 & 7 \\
\hline 1 Akzeptanz von Laufbahnmodellen & -- & & & & & \\
2 Alter & $.22^{* *}$ & -- & & & & \\
3 Geschlecht & $.24^{* * *}$ & $.32^{* * *}$ & -- & & & \\
4 Unterrichtsstufe & $.13^{*}$ & $.20^{* *}$ & $.46^{* * *}$ & -- & & \\
5 Beruflicher Ehrgeiz & $-.12^{*}$ & $-.12^{*}$ & .07 & -.09 & -- & & \\
6 Autonomie-Paritäts-Muster & $.33^{* * *}$ & $.24^{* * *}$ & $.28^{* * *}$ & $.19^{* *}$ & $-.16^{* *}$ & -- & \\
7 Kollektive SW-Überzeugungen & $-.13^{*}$ & .02 & .05 & .01 & -.01 & $-.18^{* *}$ & -- \\
\hline
\end{tabular}

Anmerkungen: Spearman's rho für $\mathrm{r}_{12} ; \mathrm{r}_{15} ; \mathrm{r}_{16} ; \mathrm{r}_{17} ; \mathrm{r}_{23} ; \mathrm{r}_{24} ; \mathrm{r}_{35} ; \mathrm{r}_{36} ; \mathrm{r}_{37} ; \mathrm{r}_{45} ; \mathrm{r}_{46} ; \mathrm{r}_{47}$

Phi-Koeffizient für $\mathrm{r}_{13} ; \mathrm{r}_{14} ; \mathrm{r}_{34}$

Pearson's $r$ für alle anderen Korrelationen.

,Akzeptanz von Laufbahnmodellen' codiert als 1 = ja, 2 = nein.

,Alter' codiert 23-61.

,Geschlecht' codiert als 1 = weiblich, 2 = männlich.

,Unterrichtsstufe' codiert als 1 = Primarstufe, 2 = Sekundarstufe I.

Alle anderen Variablen codiert 1-5.

${ }^{*} \mathrm{p}<.05 .{ }^{* *} \mathrm{p}<.01 .{ }^{* * *} \mathrm{p}<.001$. 
Die Prämisse logistischer Regressionen, die Abwesenheit von Multikollinearität bei den unabhängigen Variablen fordert, wird in Bezug auf die Variablen «Geschlecht» und «Unterrichtsstufe» verletzt, weshalb eine der beiden Variablen nicht ins Regessionsmodell aufgenommen wird. Die hohe Korrelation zwischen Geschlecht und Unterrichtsstufe ist auch in der Population gegeben und entspricht insofern den realen Gegebenheiten in der Volksschullehrerschaft. Da das Geschlecht deutlich höher mit der Akzeptanz von Laufbahnmodellen korreliert als die Unterrichtsstufe (vgl. Tabelle 2), wird die unabhängige Variable «Unterrichtsstufe» nicht in das Regressionsmodell eingeführt. Es werden also nur fünf Prädiktorvariablen berücksichtigt (vgl. Tabelle 3).

Tabelle 3: Binär logistische Regression: Kriteriumsvariable "Akzeptanz von Laufbahnmodellen»; Methode: Einschluss

\begin{tabular}{llccc}
\hline & Prädikatorvariablen & \multicolumn{1}{c}{ B } & SE & Odds ratios \\
\hline 1 & Alter & 0.27 & .025 & 1.028 \\
2 & Geschlecht & $-1.523^{* *}$ & .575 & .218 \\
3 & Beruflicher Ehrgeiz & -.603 & .356 & .547 \\
4 & Autonomie-Paritäts-Muster & $1.740^{* *}$ & .554 & 5.699 \\
5 & Kollektive Selbstwirksamkeitsüberzeugungen & -.599 & 337 & .549 \\
\hline
\end{tabular}

Keine weiteren Variablen eingeführt.

Anmerkungen: Kriteriumsvariable, Akzeptanz von Laufbahnmodellen' codiert als $0=$ ja, $1=$ nein.

,Geschlecht' codiert als 0 = männlich, 1 = weiblich. Hosmer-Lemeshow-Test: $\chi^{2}(8)=5.925$; sig. $=.656$. ${ }^{*} \mathrm{p}<.05 .{ }^{* *} \mathrm{p}<.01 .{ }^{* * *} \mathrm{p}<.001$.

Das binär logistische Regressionsmodell in Tabelle 3 zeigt die hohe Bedeutung des Geschlechts und des Ausmasses, mit dem sich Lehrpersonen mit dem Autonomie-Paritäts-Muster identifizieren, für die Akzeptanz von Laufbahnmodellen. Alter, beruflicher Ehrgeiz und kollektive Selbstwirksamkeitserwartungen verlieren demgegenüber an Bedeutung. Mit dem Geschlecht «männlich» ist die Wahrscheinlichkeit, Laufbahnmodelle abzulehnen, mehr als vier Mal so hoch als mit dem Geschlecht «weiblich».6 Eine Erhöhung der Identifikation mit dem Autonomie-Paritäts-Muster um eine Einheit auf der fünfstufigen Skala erhöht die Wahrscheinlichkeit, Laufbahnmodelle abzulehnen, gar um mehr als das 5-Fache.

\section{Auswirkungen von Laufbahnmodellen}

Die insgesamt starke Akzeptanz von Laufbahnmodellen zeigt sich in den Einschätzungen von erwartbaren Auswirkungen wieder. Eine Mehrheit der antwortenden Lehrpersonen erwartet positive Auswirkungen: 84.0 \% der Lehrpersonen erwarten eine Erhöhung der eigenen Berufszufriedenheit, drei Viertel (76.1 \%) der Lehrpersonen sind der Ansicht, dass eine Einführung von Laufbahnmodellen die Attraktivität für die Wahl des Lehrberufs erhöhen würde, und zwei Drit- 
tel $(66.0 \%)$ erwarten positive Auswirkungen auf das Image des Lehrberufs. Ebenfalls zwei Drittel $(66.0 \%)$ denken, dass sich die Schul- und Unterrichtsqualität mit einer Einführung von Laufbahnmodellen verbessern liesse.

Die grössten Befürchtungen beziehen sich auf die Zusammenarbeit in den Kollegien: $16.5 \%$ der Lehrpersonen erwarten diesbezüglich negative Auswirkungen. Allerdings erwarten fast drei Mal so viele Lehrpersonen (46.2 \%) auch auf die Zusammenarbeit in den Kollegien positive Auswirkungen.

\section{Unterstützungspotential und Attraktivität von Spezial- funktionen}

Die positiven Erwartungen an Laufbahnmodelle decken sich mindestens teilweise auch mit dem Unterstützungspotential, welches die Lehrpersonen möglichen Funktionen in einem Laufbahnmodell zuschreiben.

In Bezug auf das Unterstützungspotential von möglichen Spezialfunktionen zeigt sich, dass unterrichtsperiphere Funktionen tendenziell als hilfreicher eingeschätzt werden als unterrichtsnahe. Die Lehrpersonen wurden gebeten, eine Rangreihenfolge für das Unterstützungspotential verschiedener Funktionen zu erstellen. Dabei wird der Schulleitung (50.6 \% in den ersten drei Rangplätzen), den Funktionen für organisatorische und administrative Aufgaben (35.6 \%) sowie denjenigen für schulinterne Qualitätsentwicklung (32.4\%) das grösste Unterstützungspotential zugeschrieben. Ebenfalls vergleichsweise häufig wird als unterrichtsnahe Funktion die Betreuung von Junglehrpersonen genannt (31.1\%) - eine Funktion, die allerdings bei einem grossen Teil der Lehrpersonen den eigenen Unterricht nicht tangieren würde. Eher gering wird das Unterstützungspotential bei Funktionen für spezielle fachdidaktische Fragestellungen (8.1\%), bei didaktischen (11.7\%) oder pädagogischen Themen (13.0 \%) eingeschätzt.

Im Gegensatz zum Unterstützungspotential beurteilen die befragten Lehrpersonen vorwiegend unterrichtsnahe Funktionen als für sie selbst attraktiv (vgl. Abbildung 1): Am attraktivsten sind Spezialfunktionen für die Betreuung von Junglehrpersonen $(\mathrm{M}=2.33$; $\mathrm{SD}=0.70)$, in den Bereichen erweiterter Lehr/Lernformen $(M=2.18 ; S D=0.70)$ und spezieller didaktischer Themen $(\mathrm{M}=2.16 ; \mathrm{SD}=0.69)$. Am wenigsten attraktiv ist die Funktion der Schulleitung $(\mathrm{M}=1.67 ; \mathrm{SD}=0.77)$ - diejenige Funktion also, der das grösste Unterstützungspotential zugeschrieben wird (vgl. oben). 


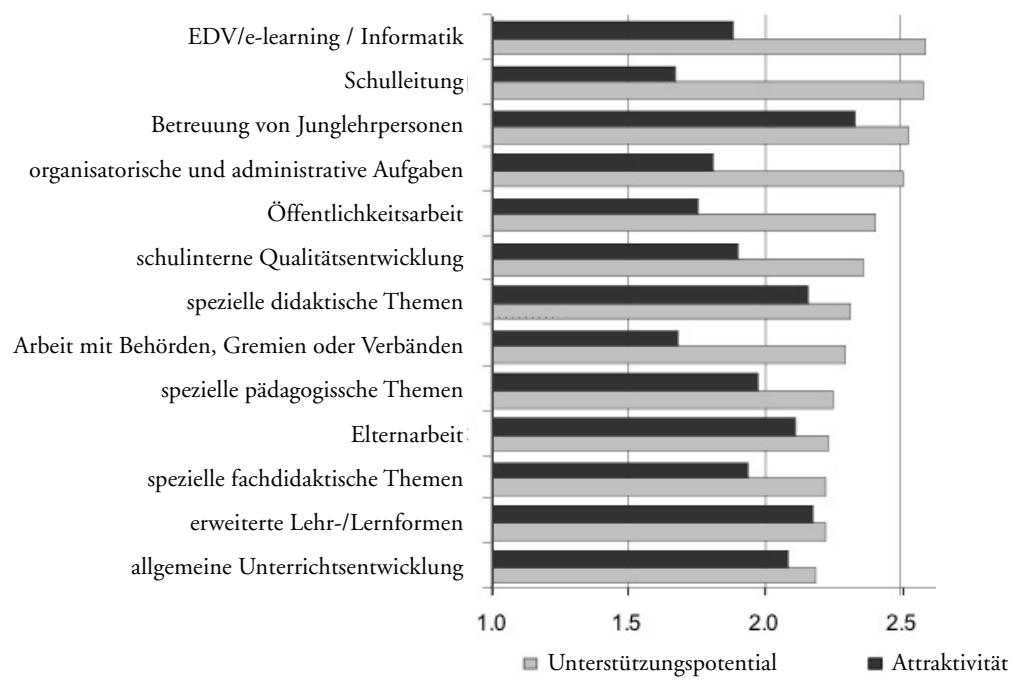

Abbildung 1: Attraktivität und Unterstützungspotential von Spezialfunktionen: Mittelwerte für die Einschätzung der Attraktivität ( $1=$ nicht attraktiv; 2 = teilweise attraktiv; 3 =attraktiv) und des Unterstützungspotentials ( $1=$ nicht hilfreich; 2 = teilweise hilfreich; 3 = hilfreich)

\section{Interpretationen der Ergebnisse}

Die sehr hohe Zustimmung zur Idee von Laufbahnmodellen bei der schriftlichen Befragung bedarf einer Interpretation. Als Schlüssel für die Erklärung der hohen Akzeptanz hat sich eine methodische Kritik erwiesen, die bei verschiedenen kommunikativen Validierungen der Ergebnisse eingebracht wurde: Die Thematik «Laufbahnmodelle» suggeriere grundsätzlich Möglichkeiten für mehr oder für gerechteren Lohn und für mehr Prestige und werde deshalb generell positiv beurteilt. Dieser Einwand kann nicht von der Hand gewiesen werden, denn die Idee von Laufbahnmodellen kann ja tatsächlich mehr Lohn und mehr Prestige beinhalten - je nach Ausgestaltung eines Modells.

\section{Wunsch nach Anerkennung}

Die Antworten der Lehrpersonen machen deshalb deutlich, dass der Wunsch nach mehr oder nach gerechterem Lohn und nach mehr Prestige, also nach mehr kommunizierbarer Anerkennung, im Lehrberuf weit verbreitet zu sein scheint. Mit dem Hinweis auf die Aufgabenvervielfältigung in den Schulen, die zu einem eigentlichen job enlargement vieler Lehrpersonen geführt hat, ist dieser Sachverhalt auch gut erklärbar: Das Engagement der Lehrpersonen für gesamtschulische Anliegen wird von institutioneller Seite bis anhin kaum anerkannt. Laufbahn- 
modelle aber stellen eine Anerkennung für diese Form der beruflichen Weiterentwicklung in Aussicht. Die vorliegenden Resultate lassen die Interpretation zu, dass eine grosse Mehrheit der Lehrpersonen - dem Grundprinzip einer Leistungsgesellschaft entsprechend - für höhere Leistungen und höhere Kompetenz auch eine entsprechende Kompensation erwartet, dass diese Lehrpersonen also auch auf in einer Leistungsgesellschaft übliche Anerkennungsformen ansprechen, dass deshalb auch die exklusive Fokussierung auf die «innere Karriere» in Frage gestellt wäre, dass also die fortlaufende Berufslaufbahn auf dem immer gleichen formalen Niveau und ohne Vorankommen für viele Lehrpersonen an Attraktivität verloren hätte. Dies kann als Anzeichen dafür gelesen werden, dass viele Lehrpersonen bereit und gewillt sind, sich auch für die neu anfallenden Aufgaben zu engagieren, ihr ursprüngliches Tätigkeitsfeld auszuweiten und dadurch Verantwortung für die Gesamtschule mit zu übernehmen, sich insofern also an einer Form von "Distributed Leadership» zu beteiligen.

Die höhere Akzeptanz bei Frauen als bei Männern unterstützt diese Interpretation: Die deutlich häufigere Präsenz von Männern in Schulleitungsfunktionen eröffnet den Lehrern auch ohne Fachlaufbahnen eine Perspektive zur beruflichen Weiterentwicklung und zur Übernahme von Verantwortung für die Gesamtschule. Lehrerinnen hingegen, die sich bis anhin tendenziell weniger für eine solche Management-Laufbahn entschieden haben, sehen in unterrichtsbezogenen Fachlaufbahnen deshalb stärker eine neue Perspektive, die es ihnen erlauben würde, sich an der Verantwortung für gesamtschulische Anliegen und damit an einem «Distributed Leadership» zu beteiligen.

\section{Hoffnung auf Entlastung}

Eine andere Erklärung für die hohe Akzeptanz von Laufbahnmodellen zeigt sich bei der Einschätzung des Unterstützungspotentials möglicher neuer Funktionen. Die deutliche Bevorzugung von unterrichtsperipheren gegenüber unterrichtsnahen Funktionen legt die Interpretation nahe, dass mit der hohen Akzeptanz von Laufbahnmodellen auch ein starker Wunsch nach oder eine grosse Hoffnung auf Entlastung im Schulalltag kommuniziert wird: Laufbahnmodelle sollten Funktionen vorsehen, welche die Lehrpersonen bei der Bewältigung der zahlreichen Aufgaben quantitativ entlasten - für viele ist es weniger dringend, eine qualitative Unterstützung für gewisse Aspekte beim eigenen Unterrichten zu erhalten.

Dieser klare Vorzug so genannter non-reform roles (Donaldson et al., 2005) also von Funktionen, die nicht auf Veränderungen in den Unterrichtstätigkeiten zielen - kann als Hinweis dafür interpretiert werden, dass das Autonomie-Paritäts-Muster vielleicht doch noch nicht so stark überwunden ist, wie dies die hohe Akzeptanz von Laufbahnmodellen vermuten liesse. Somit könnte die hohe Akzeptanz von Laufbahnmodellen auch als Ausweg aus einem Dilemma interpretiert werden: aus dem Dilemma zwischen der vorherrschenden Berufskultur und einer Überlastung durch die Aufgabenvervielfältigung. Das vorherrschende Berufsverständnis wäre somit stark vom Autonomie-Aspekt geprägt: Die Auto- 
nomie für das Kerngeschäft «Unterricht» soll möglichst nicht angetastet werden, Freiheit und Verantwortung sind für die ganze zu bewältigende Aufgabe vorgesehen. Mit der Aufforderung zur Schulentwicklung, die auf gemeinsames Lernen und geteilte Überzeugungen und Normen ausgerichtet ist, wird eine solche Berufsauffassung in Frage gestellt. Laufbahnmodelle aber, die non-reform roles vorsehen, lösen dieses Dilemma auf: Im Unterricht bleibt die Autonomie gewahrt, Lehrpersonen können hier alle Aufgaben, für die sie zuständig sind, in Eigenverantwortung leisten, für die anderen Aufgaben werden Spezialfunktionen geschaffen.

Diese Ergebnisse, die auf eine eher zurückhaltende Einschätzung des Potentials von reform-roles hinweisen, machen deutlich, dass Lehrpersonen keinen «Superhero» in ihren Schulen wollen, keinen «know-it all». Vielmehr scheinen Lehrpersonen für die Kernaufgaben eine Form von Leadership "of many rather than the few» zu bevorzugen. Dies weist auf das Potential hin, Leadership in der Organisation breiter zu verteilen, so dass sich mehrere Lehrpersonen über Formen von "Distributed Leadership» für gesamtschulische Anliegen zu engagieren bereit und gewillt sind - wenn denn die Anerkennung dafür geleistet wird.

Mit dieser Interpretation stellen sich Fragen nach den Möglichkeiten von Leadership der Schulleitungen. Die vorliegenden Daten erlauben den Schluss, dass in der vorherrschenden Berufskultur differenzierte Rollen und damit Formen von "Distributed Leadership» sehr wohl akzeptiert und teilweise sogar erwünscht sind - wenn sie denn Entlastung bei der Bewältigung der eigenen alltäglichen Arbeit versprechen und wenn sie über keine Linienvorgesetzte einen Eingriff in die eigene Unterrichtstätigkeit befürchten lassen. Wie weit unter dieser Prämisse jedoch ein eigentlich pädagogisches Leadership in den Schulen gelebt werden kann, ist in Frage zu stellen. Die vorliegenden Daten weisen eher darauf hin, dass die Lehrpersonen gewillt sind, im Kernbereich selbst die Verantwortung zu behalten. Damit scheinen sie sich einerseits am Leadership in der eigenen Schule beteiligen zu wollen, zeigen andererseits aber auch die Grenzen eines konventionellen Führungsverständnisses auf.

\section{Anmerkungen}

1 Im Kommentar dazu heisst es u.a.: «Die Flexibilität in der innerschulischen Aufgabenverteilung und die Begünstigung individueller Entwicklungen und Veränderung im Beruf sind wichtig. Das hat mit Karrieredenken nichts zu tun, sondern

- mit der vollen ,betrieblichen' Nutzung besonderer individueller Kompetenzen und Interessen zur Erhaltung und Entwicklung der Schulqualität;

- mit der Glaubwürdigkeit eines Berufs, welcher mit Lernen und damit mit persönlicher Entfaltung und Weiterentwicklung zu tun hat;

- mit der Gesundherhaltung im Beruf, der Aufrechterhaltung der Motivation, mit dem Vermeiden von Ausbrennen und Stagnation;

- mit der Einladung, die vor allem in späteren beruflichen Phasen auftretenden Diversifikationsinteressen nicht nur ausserhalb der Schule, sondern auch in schulischen Sonderaufgaben zu verwirklichen.» 
2 Die Begriffe «Rolle» und «Funktion» werden im vorliegenden Beitrag synonym verwendet, da sie sich nur aus theoretischer Perspektive unterscheiden, indem die Bezeichnung «Rolle» die Sicht der Mitarbeitenden und die Bezeichnung «Funktion» diejenige der Organisation ausdrückt.

3 Es könnten hier zahlreiche weitere Beispiele bezüglich der Entwicklung des Lehrberufs in Richtung neuer Positionen für Lehrpersonen mit besonderen Qualifikationen, Verantwortungen und Anerkennung angeführt werden, u.a. die Auseinandersetzungen im Zusammenhang mit der Einführung der Schulleitungen oder die Kontroversen um die lohnwirksame Beurteilung von Lehrpersonen. Dies kann jedoch im hier zur Verfügung stehenden Rahmen nicht geleistet werden.

4 Mit den diesem Muster entsprechenden informellen Normen, dass kein Erwachsener in den Unterricht der Lehrpersonen eingreifen soll (Autonomie) und dass alle Lehrpersonen als Gleichberechtigte zu betrachten und zu behandeln seien (Parität), reagierte der Berufsstand auf die mit der Modernisierung einhergehende zunehmende Bürokratisierung des Lehrberufs und verschaffte den einzelnen Lehrpersonen jene individuelle und spontane Freiheit, die sie zur Erfüllung ihres Berufsauftrages brauchen. «In soziologischer Terminologie ausgedrückt: Das Autonomie-Paritäts-Muster, mit der Isolierung als Grundstruktur, ist das funktionale Äquivalent der klar definierten Privilegien eines Arztes, Architekten oder erfolgreichen Künstlers. Es bewirkt eine Restriktion des Einflusses der formellen und bürokratischen Ordnung, die - auf dem Papier - die Struktur des öffentlichen Schulwesens [...] verkörpert» (Lortie, 1972, S. 47).

5 Itemformulierung: «Im Lehrberuf gibt es bis anhin verhältnismässig wenig Aufstiegsmöglichkeiten. Lehrpersonen treten nach ihrer Grundausbildung in den Unterrichtsberuf ein und bleiben oft Lehrerin oder Lehrer bis zu ihrer Pensionierung. Für eine berufliche Entwicklung, die auch eine Veränderung der Funktion beinhaltet, mussten Lehrpersonen bis anhin ihren Beruf verlassen. Es ist nun denkbar, auch für den Lehrberuf - wie in anderen Berufsfeldern üblich - berufliche Aufstiegsmöglichkeiten zu schaffen. Solche Laufbahnmodelle könnten den Lehrpersonen beispielsweise erlauben, sich für zusätzliche Positionen zu qualifizieren. Diese Positionen würden eine neue Stellung mit erweiterter Verantwortung mit sich bringen, beispielsweise für Coaching von Junglehrpersonen oder für Beratung von Kolleginnen und Kollegen bei der Einführung von neuen Lehr-/Lernformen.

Finden Sie persönlich die Einführung von solchen Laufbahnmodellen grundsätzlich sinnvoll?»

6 Odds ratios $=.218(1 / 0.218=4.587)$

\section{Literatur}

Ammann, T. (2004). Zur Berufszufriedenheit von Lehrerinnen. Erfahrungsbilanzen in der mittleren Berufsphase. Bad Heilbrunn: Verlag Julius Klinkhardt.

Baumgartner, V. \& Kassis, W. (2001). Erhebung zum Bedarf an Zusatzausbildungen für Funktionen in Bildungssystemen im Auftrag der Arbeitsgruppe Lehrerbildung der EDK, Unterlagen zur Tagung vom 7. Juni 2001 in Basel. Version vom 6. Juni 2001.

Bennett, N., Wise, C., Woods, P. \& Harvey, J. A. (2003). Distributed Leadership. London: National College for School Leadership.

Bessoth, R. \& Landolt, H. (1997). Organisations-Klima-Instrument für Schweizer Schulen (OKI-CH, Version 8.5): Ein Leitfaden zur Schulentwicklung mit Fragebogen und WINDOWS-Diskette. Aarau: Sauerländer.

Bucher, B. \& Nicolet, M. (2003). Leitbild Lehrberuf. Studien und Berichte 18a. Bern: EDK (Schweizerische Konferenz der kantonalen Erziehungsdirektoren).

Buholzer, A. (2000). Das Innovationsklima in Schulen. Aarau: Sauerländer.

Bundesamt für Statistik. (2000). Lehrkräfte 1998/99. Neuenburg: Bundesamt für Statistik. 
Domsch, M. E. (1994). Fachlaufbahn - ein Beitrag zur Flexibilisierung und Mitarbeiterorientierung der Personalentwicklung. In M. E. Domsch \& S. H. A. Siemers (Hrsg.), Fachlaufbahnen (S. 5-21). Heidelberg: Physica.

Donaldson, M. L., Kirkpatrick, C. L., Marinell, W. H., Steele, J. L., Szczesiul, S. A. \& Johnson, S. M. (2005). "Hot shots» and "principal's pets»: How colleaugues influence second-stage teachers' experience of differentiated roles. Paper presented at the American Educational Research Association annual conference, Montreal (Can.).

EDK. (1978). Auswertung der Vernehmlassung zum Bericht «Lehrerbildung von morgen». EDK Informationsbulletin 12a. Genf: EDK.

Education Writers Association. (2002). Searching for a superhero: Can principles do it all? EWA Special Report. Washington: EWA.

Ender, B. \& Strittmatter, A. (2001). Personalentwicklung als Schulleitungsaufgabe. Innsbruck: Studienverlag.

Fiechter, U., Stienen, A. \& Bühler, C. (2004). Zukünftige Lehrpersonen: Berufswahl als pragmatisch orientierte Individualisierung. Eine Studie zur Situation im Kanton Bern. Bern: Institut für Lehrerinnen- und Lehrerbildung Marzili.

Füchtner, S. (1998). Karriereplanung im Wandel. Personal: Zeitschrift für Human Ressource Management, 12 (50), 602-607.

Harris, A. (2004). Distributed leadership and school improvement: Leading or misleading? Educational Management Administration \& Leadership, 32 (1), 11-24.

Harris, A. \& Lambert, L. (2003). Building leadership capacity for school improvement. Milton Keynes: Open University Press.

Hearvey-Beavis, O. (2003). Performance-Based Rewards for Teachers: A Literature Review. Paris: OECD (Organisation for Economic Cooperation and Development).

LCH. (1999). LCH-Berufsleitbild, LCH-Standesregeln: Verabschiedet von der LCH-Delegiertenversammlung am 19. Juni 1999.

Leutwyler, B., Sieber, P. \& Diebold, M. (2005). Untersuchung zur Akzeptanz von Laufbahnmodellen im Lehrberuf. Chancen- und Risikoeinschätzung. Zug: PHZ Zug / Institut für internationale Zusammenarbeit in Bildungsfragen.

Lortie, D. C. (1972). Team Teaching. Versuch der Beschreibung einer zukünftigen Schule. In H.-W. Dechert (Hrsg.), Team Teaching in der Schule (S. 37-76). München: Piper.

Lortie, D. C. (1975). Schoolteacher. A Sociological Study. Chicago: The University of Chicago Press.

Muijs, D. \& Harris, A. (2003). Teacher leadership: A review of the literature. Educational Management Administration \& Leadership, 31 (4), 437-439.

Müller, F. (1975). Lehrerbildung von morgen. Grundlagen, Strukturen, Inhalte. Bericht der Expertenkommission "Lehrerbildung von morgen" im Auftrag der Schweizerischen Konferenz der kantonalen Erziehungsdirektoren. Hitzkirch: Comenius-Verlag.

Peske, H. G., Liu, E., Johnson, S. M., Kauffmann, D. \& Kardos, S. M. (2001). The next generation of teachers: Changing conceptions of a career in teaching. Cambridge, MA: Harvard Graduate School of Education.

Rolff, H.-G. (1996). IFS-Schulbarometer. Lehrerinnen- und Lehrerteil. Dortmund: Institut für Schulentwicklungsforschung.

Santiago, P. (2002). Teacher demand and supply: Improving teaching quality and addressing teacher shortages. A literature review and a conceptual framework for future work. Paris: OECD.

Schaarschmidt, U. \& Fischer, A. (1996). AVEM - Arbeitsbezogenes Verhaltens- und Erlebensmuster. Frankfurt a.M.: Swets \& Zeitlinger.

Schacter, J., Schiff, T., Thum, Y. M., Fagnano, C., Bendotti, M., Solmon, L., Firetag, K. \& Milken, L. (2003). The impact of the teacher advancement program on student achievement, teacher attitudes and job satisfaction. Santa Monica, CA: Milken Familiy Foundation. 
Schwarzer, R. \& Jerusalem, M. (1999). Skalen zur Erfassung von Lehrer-und Schülermerkmalen. Dokumentation der psychometrischen Verfahren im Rahmen der Wissenschaftlichen Begleitung des Modellversuchs «Selbstwirksame Schulen». Berlin: Freie Universität Berlin.

Seifert, K. H. (1992). Berufswahl und Laufbahnentwicklung. In D. Frey, C. G. Hoyos \& D. Stahlberg (Hrsg.), Angewandte Psychologie. Ein Lehrbuch (S. 187-204). Weinheim: Psychologie Verlags Union.

Spillane, J. (2005). Distributed leadership. In M. J. Coles \& G. Southworth (Hrsg.), Developing leadership: Creating the schools of tomorrow. Buckingham: Open University Press.

Schlagworte: Lehrberuf, Leadership, Berufsbild, Berufslaufbahn, Professionalität

\section{La profession d'enseignant en mutation? Des limites du leadership.}

\section{Résumé}

Le transfert de responsabilité aux écoles observable ces dernières années est lié à l'introduction, dans la plupart des cantons suisses, de directions d'établissement (Schulleitungen). Ceci a rendu possible d'introduire un profil d'enseignant qui caractérise la formation professionnelle du personnel enseignant dans d'autres pays depuis longtemps: une carrière professionnelle. Cependant, en Suisse, les tentatives d'établir de nouvelles possibilités de promotion professionnelle ou de différenciation de rôles au sein de la profession d'enseignant ont rencontré jusqu'à présent de fortes résistances de la part du corps enseignant. Par conséquent, ni les associations professionnelles ni les autorités ne se sont sérieusement penchées sur ces concepts. Dans le contexte du changement des conditions cadres mentionné ci-dessus, un projet de recherche de l'IZB (Institut de Coopération Internationale en Education) a étudié comment les différents groupes concernés jugent l'introduction de modèles de carrière aujourd'hui et comment les résistances observées dans le passé continuent d'être répandues. Des enseignants de différents niveaux d'école obligatoire ont été interrogés dans quatre cantons suisses allemands à l'aide d'un questionnaire. Par la suite, des interviews plus approfondies ont été réalisées avec des enseignants, des membres de l'administration et des associations professionnelles. Les résultats de l'enquête montrent que, d'une part, une majorité écrasante des enseignants interrogés accordent un soutien de principe à l'introduction d'un modèle de carrière. D'autre part, il ressort qu'un tel modèle suscite des attentes très différentes. Les résultats soulèvent la question de savoir à quel point le leadership pédagogique est envisageable dans la culture professionnelle suisse actuelle. Ils montrent également la volonté du personnel enseignant luimême de participer activement au leadership pédagogique.

Mots clés: Profession d'enseignant, leadership, profil professionnel, carrière professionnelle, professionnalité 


\section{Sta cambiando la professione d'insegnante? Sui limiti della leadership.}

\section{Riassunto}

La consegna di responsabilità al mondo della scuola che si è osservata negli anni passati è da mettere in relazione, nella maggior parte dei cantoni svizzeri, con l'introduzione delle direzioni scolastiche (Schulleitungen). Con questo provvedimento è diventato possibile un approccio a ciò che in altri paesi già da tempo caratterizza il profilo professionale dell'insegnante: una carriera professionale. Tuttavia, in Svizzera i tentativi di stabilire delle ulteriori possibilità di promozioni professionali o di differenziazione dei ruoli nella professione d'insegnante, si sono scontrati fino ad ora con le forti reazioni di difesa da parte del corpo insegnante e, di conseguenza, hanno tenuto lontano i gruppi professionali e le autorità dall'interessarsi seriamente a queste idee. Sullo sfondo di condizioni settoriali mutate, un progetto di ricerca dell'IZB ha investigato su come oggi differenti gruppi di pressione considerano l'introduzione di un modello carrieristico e su come le reazioni di difesa osservate in passato si siano allargate oggi. Attraverso un questionario, sono stati interrogati gli insegnanti di differenti livelli di scuola pubblica in quattro cantoni della Svizzera tedesca. Inoltre sono state effettuate delle interviste più approfondite con insegnanti e con rappresentanti dell'amministrazione e di associazioni. I risultati dello studio mostrano che, da un lato, una sovrastante maggioranza degli insegnanti interrogati sostiene in principio un'introduzione di un modello carrieristico, ma che, dall'altro, i modelli carrieristici sono da mettere in relazione con speranze profondamente differenti. Questi risultati sollevano il problema di valutare fino a che punto le leadership pedagogiche possono essere vissute nella cultura dominante della professione, e mostrano come il personale insegnante stesso è assolutamente volitivo a partecipare attivamente alla leadership.

Parole chiave: professione dell'insegnante, leadership, profilo professionale, carriera professionale, professionalità

\section{Changes in the field of teaching profession? The limits of leadership.}

\section{Summary}

The transfer of responsibility to schools that could be observed in recent years has gone hand in hand in most Swiss cantons with the introduction of school management boards (Schulleitungen). This has created opportunities for a professional career for teachers, which has long existed in other countries. However, previous efforts to establish career opportunities or role differentiations in 
Switzerland have encountered resistance from teachers and, as a consequence, neither professional associations nor public authorities dealt seriously with these concepts. In the context of the above-mentioned new framework, a research project at IZB (Institute for International Cooperation in Education) studied how the groups involved position themselves today with respect to the introduction of career schemes and to what extent the former resistance is still widespread. Teachers from different levels in compulsory schools in four Germanspeaking cantons were interviewed using questionnaires. Furthermore, in-depth interviews were conducted with teachers as well as with representatives of administrations and professional associations. The results of the study show that an overwhelming majority of teachers is in principle in favour of the introduction of career schemes. However, the results also show that very different expectations are attached to these schemes, raising the question as to what extent pedagogical leadership is feasible in the existing professional culture in Switzerland. Finally, the results reveal that teachers are in principle willing to take on pedagogical leadership.

Keywords: Teacher, leadership, professional profile, career, professionalism 